\title{
Miedo a volar: Componentes y formas de afrontamiento de un grupo de patagones que viven en aislamiento relativo
}

\section{Fear of flying: Components and forms of coping with a group of Patagonians living in relative isolation}

\author{
José Luis Mimica \\ Gonzalo Castillo \\ Claudia Estrada \\ Escuela de Psicología, Universidad de Magallanes, Chile \\ Rec (27 de noviembre de 2017) Acept (5 de julio de 2018)
}

\begin{abstract}
Resumen
El presente estudio trata del temor a volar, las expectativas de peligro y las estrategias de afrontamiento que las personas dicen utilizar al momento de volar en avión. Un total de 287 participantes, habitantes de una zona aislada geográficamente, que utilizan habitualmente el avión, fueron medidos en las variables ya señaladas. El muestreo fue no probabilístico accidental y el diseño utilizado fue no experimental transversal correlacional. Los principales resultados indican que las personas dicen temer principalmente a situaciones que provocan en ellos la pérdida del control emocional aunque sus estrategias de afrontamiento más comunes suelen estar orientadas a la resolución de problemas. Estas estrategias, orientadas a recuperar simbólicamente el control psicológico, no apuntan al afrontamiento de las emociones. También se describen algunas diferencias en el uso de estas estrategias según género. Se discuten estos resultados en términos de su sincronía con los estudios previos y sus potenciales implicancias para los actuales programas terapéuticos de intervención de dicho problema.

Palabras clave: Miedo a volar, Estrategias de afrontamiento, Expectativas de peligro
\end{abstract}

\begin{abstract}
This study is about the fear of flight, the danger expectations and the coping strategies that people say to use when flying on an airplane. A total of 287 participants, people from a graphically isolated zone that usually use planes, were measured according to the already mentioned variables. The sampling was not accidental probabilistic and the designed used was not experimental transversal correlational. The principal results indicate that people say to be afraid principally of situations that cause in them the loss of the emotional control, although, their most common coping strategies use to be oriented to affront anxiety. This strategy, aimed to regain the psychological control symbolically, does not point to the emotions coping. Also, some differences in the use of these strategies according to gender are described. These results are discussed regarding its timing with previous studies and their potential implications for current therapeutic intervention programs for this problem.

Keywords: Fear of flight, Coping strategies, Danger expectations.
\end{abstract}




\section{Introducción}

El miedo es una característica temprana en la humanidad, que comparte con los otras especies, y que ha tenido como finalidad la adaptación y la supervivencia (Darwin, 1872). Este miedo, que resulta tan útil para los animales porque se activa frente a episodios de peligro que requieren reacciones fisiológicas para enfrentarlo, en los humanos puede ser activado frente a situaciones que son percibidas como peligrosas aunque no lo sean en la realidad (Gray, 1971). Reiss (1980) propone diferenciar entre lo que denomina "expectativas de peligro" y "expectativas de ansiedad". Mientras que las primeras serían las que motivan a evitar estímulos asociados a un posible daño procedente del ambiente externo, las segundas motivarían al individuo a evitar estímulos asociados a posibles daños o malestares internos. De acuerdo con el DSM-IV (APA, 1995) estos sentimientos de ansiedad pueden hacer que la persona adquiera fobias, término proveniente del griego y cuya aplicación está vinculada al miedo. Para estar en presencia de un cuadro fóbico se tienen que registrar temores irracionales o incluso aversiones obsesivas hacia objetos, situaciones, seres vivos, entre otros.

Un escenario que es fuente de temor en la actualidad, y que incluso ha llegado a considerarse como una de las más importantes fobias específicas, es aquella que se produce por la experiencia de volar en avión (Cummings, 1989). La frecuencia de esta experiencia ha aumentado considerablemente los últimos años. Según la revista de viajes "Travel 3Sixty" (revista de la aerolínea Air Asia), se estima que más de mil millones de personas al año usan este medio de transporte en el mundo. Respecto al tráfico nacional, la empresa privada SCL Terminal Aéreo Santiago S.A informó que durante el 2012 se obtuvo un incremento de un $19 \%$ en relación al 2011, esto se observa en el aumento de 6.232.133 pasajeros a un total de 7.414 .698 , mientras que en Magallanes son cerca de trescientas mil las personas que usan este medio de trasporte durante un año. Aunque volar muchas veces se asocia a experiencias positivas tales como las vacaciones, y se le reconoce como un medio seguro que ahorra tiempo y aporta comodidad, son numerosas las personas que presentan algún tipo de miedo asociado al volar en avión. Se calcula que, en sus diversos grados, esta fobia afecta aproximadamente a una cuarta parte de la población general (Greco, 1989; Van Gerwen, Spinhoven, Diekstra \& Van Dyck, 1997).

Aunque para la mayoría de las personas con temor a volar esta experiencia no resulta invalidante, es decir, no le impide viajar en avión, si representa una experiencia de temor que se asocia a una serie de creencias y de acciones que buscan mantener el control psicológico de las personas. En relación a lo anterior aparecen las estrategias de afrontamiento (EA) que son definidas como aquellos recursos cognitivos y conductuales que ayudan a superar las demandas psicológicas que surgen al momento en que una persona se ve enfrentada a una situación en la que sus recursos personales se ven sobrepasados (Lazarus \& Folkman, 1986). En este sentido, el afrontamiento del estrés sería el resultado del encuentro entre la persona, sus recursos personales, personalidad, etc. y una situación potencialmente estresante. Esta necesaria interacción entre ambos componentes estipulará si una determinada experiencia resultará o no estresante para una persona específica. Si bien muchos autores le dan énfasis al hecho de que son las características individuales y la personalidad factores básicos en su génesis (McCrae \& Costa, 1986) también hay muchos otros que explican que la teoría del aprendizaje social es un eje fundamental para adquirir formas de afrontamiento (Bandura \& Walters, 1974).

Desde la perspectiva del modelo transaccional, Lazarus (1994) plantea que ante una situación estresora se tienen que tener en cuenta dos aspectos fundamentales. Por un lado, la valoración que el individuo hace sobre una situación potencialmente abrumadora o estresante y, por otro, las emociones relacionadas a éstas. El tipo de afrontamiento que la persona activa ante una situación puede estar orientado de dos maneras: (1) Guiado a enfrentar la situación de manera directa, por ejemplo buscándole una solución, o evitándola y (2) Focalizado en las emociones que dicha situación genera, como pueden ser el enojo, miedo, tristeza, etc. (Endler \& Parker 1990). Podemos hacer frente a una situación compleja aplicando una o más estrategias, ya que lo importante no es cuál ni cuántas utilicemos, si no la efectividad de éstas (Roussi \& Vassilaki, 2001). Cuando las personas no logran afrontar situaciones de estrés de manera adecuada, se tiende a la desadaptación y el estrés puede pasar de manifestarse de manera aislada a tomar características crónicas (Escamilla, Rodríguez \& González, 2009).

$\mathrm{Si}$ bien en la actualidad existen variados modelos que intentan ordenar más o menos las diferentes estrategias de afrontamiento, hay consenso en que el postulado por Lazarus \& Folkman (1986) y llamado WOC (Ways of Coping) es el que las reúne de forma más completa (Vázquez, Crespo, López \& Ring, 2003). En base al modelo anteriormente mencionado se desarrolló un instrumento para medirlas llamado CAE (Cuestionario de afrontamiento del estrés); (Sandín \& Chorot, 2006). La escala está conformada de la siguiente forma: Se presentan siete formas de afrontamiento, las cuales están dividas en dos categorías. La primera reúne a las estrategias que se encuentran focalizadas en el afrontamiento directo de la dificultad, llamada por algunos “afrontamiento racional” (Rogers, Jarvis \& Najarian, 1993) o "afrontamiento focalizado en la tarea" (Lazarus \& Folkman, 1986). La segunda categoría ordena las estrategias que están centradas en el "control emocional", llamada por algunos "Afrontamiento centrado en la emoción" (Lazarus \& 
Folkman, 1986) o "afrontamiento de tipo pasivo" (Billings \& Moos, 1981). Dentro de la primera categoría encontramos tres estrategias, la primera llamada "Focalizado en la solución de problemas" que intenta afrontar la dificultad mediante acciones directas que apuntan a resolver la situación que provoca el estrés. En segundo lugar encontramos a la "Evitación" que tiene relación con el uso de pensamientos irreales improductivos (p. ej., «ojalá hubiese desaparecido esta situación») o del uso de estrategias como beber algo y tomar algún tipo de medicamento contra la ansiedad. Como tercera y última estrategia relacionada al control racional de la situación encontramos a la "Reevaluación Positiva", en la que la persona intenta centrar sus pensamientos en algo positivo o beneficioso relacionado a la situación que lo aqueja. Como segunda categoría encontramos a las estrategias que están centradas en el control de las emociones, en donde primero encontramos la "Expresión Emocional Abierta", la cual se pone en acción cuando el sujeto es capaz de expresarse emocionalmente mediante palabras o gestos. Le sigue la "Religión", la cual busca centrar la emocionalidad en aspectos asociados a creencias religiosas arraigadas en la persona, de forma que pueda buscar un soporte en ello. También se encuentra la "Autofocalización negativa", la cual tiene vínculo con la resignación o indefensión que las personas pueden experimentar al momento de vivir una situación complicada. Finalmente encontramos al "apoyo social", el cual busca la salida al problema mediante el resguardo en personas cercanas, ya sea emocionalmente o como medio para encontrar ideas que den solución a la situación en sí misma.

Este modelo ha sido utilizado para comprender diferentes situaciones de temor o estrés. En el caso del presente estudio, el miedo a volar en avión se vincula necesariamente a las expectativas de peligro que esta situación comporta. Las expectativas de peligro (EP) pueden ser definidas como estimaciones que hacen los sujetos sobre señales del mundo externo que les indican que una situación puede ser más o menos dañina para sí mismo. Dependiendo de las características de la situación ansiógena, las expectativas de peligro pueden ser más o menos específicas. En el caso del miedo a volar, en comparación con otras situaciones de orden más cotidiano, las expectativas son más fáciles de delimitar (Tortella-Feliu \& Rubí, 2000). En este sentido, se esperaría que las estrategias de afrontamiento para este tipo de temor fueran más del orden del enfrentamiento y resolución de la fuente de temor.

La investigación respecto a las variables relevantes al momento de establecer el uso de las EA señala que existen una serie de ellas que resultan de interés. Según el género, estudios muestran que las mujeres presentan mayor uso de estrategias como el "apoyo social", prefiriendo compartir sus problemas con otros, controlando así sus emociones negativas (Cohen \& Wills, 1985). Además, también mues- tran un mayor uso de las estrategias relacionadas con la religiosidad y, en general, toda estrategia que implica la regulación emocional (Malcarne \& Fondacaro, 1988). En el caso de los varones, las investigaciones muestran que éstos prefieren negar la existencia de problemas y buscan distracciones para así superar momentos complejos, utilizando preferentemente la "evitación" (Cohen, Sherrod \& Clark, 1986). La edad también ha mostrado una asociación diferencial con el uso de las EA relacionadas con la etapa vital en que cada persona se sitúa. Por ejemplo, en el caso de los adultos mayores se presentan mayores focos de estrés asociados a cambios físicos, psicológicos, familiares, sociales y laborales. Como consecuencia, suelen utilizar una mayor variedad de recursos aunque su efectividad depende de su desarrollo (Gonzalez-Celis \& Padilla, 2006). Desde la perspectiva del nivel socioeconómico también se han encontrado diferencias significativas. Investigaciones muestran que frente a situaciones complejas las personas provenientes de niveles socioeconómicos bajos usan menor variedad de estrategias de afrontamiento, además de utilizarlas de forma poco adaptativa (San Martín, 2012). Aunque existen numerosos otros estudios que vinculan a las estrategias de afrontamiento con diversas variables, existe consenso general sobre que un punto central en la utilidad de estas herramientas está en la combinación entre las propias características personales y la evaluación de la situación. De esta forma, el afrontamiento efectivo se puede observar desde la perspectiva del mundo interno de la persona por un lado y su relación con el mundo externo por otro (Contini, Coronel, Levin \& Estevez, 2003).

La escasa atención prestada a la fobia o el miedo a volar no se justifica si se tiene en cuenta, como hemos mencionado anteriormente, que ha sido considerada como la más común de las fobias específicas (Cummings, 1989). La mayoría de los estudios al respecto giran en torno a la aplicación de tratamientos generales, con un pobre desarrollo de investigación orientada a comprender en profundidad el fenómeno y sus posibles consecuencias (Bornas \& Torte1la-Feliu, 1995). A finales de los noventa, las investigaciones se centraron en 3 aspectos principalmente: la determinación de las tasas de prevalencia, las dimensiones del miedo a volar y el análisis de sus vías de adquisición (Curtis, Magge, Eaton, Wittchen \& Kessler, 1998). Van Gerwen, Spinhoven, Diekstra \& Van Dyck (1997) señalan que la prevalencia del miedo a volar, en sus distintos grados, está entre el 10\% y el $40 \%$ de la población general en países industrializados. Con respecto al análisis de las dimensiones, el estudio de Howard, Murphy \& Clarke (1983) fue el primero que se ocupó del tema, utilizando diversos instrumentos de evaluación en las personas que acudían a tratamiento por esta problemática, pudiendo así identificar algunas categorías o dimensiones del miedo a volar, como por ejemplo el miedo a tener un accidente, miedo a experimentar sensaciones 
desagradables y miedo a estar en una situación en la que no se tiene control. Referente a las vías de adquisición, un estudio realizado en 84 personas con miedo a volar por Sosa, Capafóns \& Viña (1993) informó que el 53\% de los sujetos atribuían la instauración del problema a una experiencia directa, el $11 \%$ a una experiencia indirecta y el $34,5 \%$ no podían especificarlo. Aunque existe numerosa literatura respecto a la eficacia de tratamientos para enfrentar el miedo a volar, los estudios científicos que se interesan en los mecanismos psicológicos que las personas utilizan para enfrentarlo son escasos sino inexistentes.

El presente estudio busca ampliar el conocimiento existente sobre el mencionado problema, abordando las estrategias de afrontamiento que se utilizan para aplacar el miedo producido, las expectativas de peligro que presentan las personas cuando se les vincula al volar en avión, además de situaciones que podrían resultar de utilidad para facilitar el viaje en dicho medio de transporte. El interés en la descripción de este fenómeno radica tanto en establecer parámetros generales de la forma en que se presenta, como en su potencial aplicabilidad para el desarrollo de entornos contextuales que faciliten una experiencia de volar menos ansiógena. En consideración que los participantes del estudio pertenecen a una zona de aislamiento relativo, en la que el uso del medio de transporte aéreo no sólo es necesario sino que común, se espera aportar información que contribuya al su bienestar cotidiano.

\section{Método}

\section{Participantes}

Participaron 287 personas, provenientes de la región de Magallanes (rango de edad entre los 18 y los 83 años, $\mathrm{M}=26.3$ ), quienes fueron seleccionados mediante muestreo no aleatorio accidental. Un $56.8 \%$ de la muestra fue de género femenino y el $43.2 \%$ restante de género masculino. El criterio de inclusión fue el de haber viajado en avión en una o más oportunidades durante su vida.

\section{Diseño}

Se utilizó un diseño no experimental transversal descriptivo - correlacional.

\section{Procedimiento}

Los instrumentos fueron auto-aplicados de forma individual. Se les expuso la finalidad del estudio y se les invitó a leer y firmar un consentimiento informado. Finalmente se recibió el cuestionario con sus correspondientes respuestas, para luego aclarar dudas y agradecer su participación.

\section{Instrumentos}

- Escala Miedo a Volar: Se utilizó para determinar cuáles son las situaciones relacionadas con viajar en avión a las que las personas le tienen o no miedo. El instrumento utilizado es una adaptación del cuestionario Fear Of Flying Scale de Haug (1987) hecha por Sosa, Capafóns, Vina \& Herrero (1995). Está constituido por 21 afirmaciones que se responden sobre escalas Likert. Puntajes altos indican presencia de miedo a volar. La confiabilidad para esta muestra fue de .97 (Alfa)

- Escala de expectativas de peligro y ansiedad para el miedo a volar: Se usó para medir las creencias acerca de aquello que podría "resultar mal" durante un viaje en avión. Referido primero a pensamientos catastróficos que pueden pasar por la mente de una persona que viaja en avión y, por otro lado, enfocado en encontrar síntomas físicos relacionados a la ansiedad al momento de viajar. Este instrumento es una adaptación de la Expectative Scale For Fear Of Flying de Gursky y Reiss (1987) hecha, al igual que el instrumento anterior, por Sosa, Capafóns, Viña \& Herrero (1995). Está constituido por 19 ítems que se responden sobre una escala de Likert. La confiabilidad para esta muestra fue de .95 (Alfa).

- Estrategias de Afrontamiento de estrés frente al miedo a volar: Que intenta medir cuáles son las acciones que realizan las personas para enfrentar su miedo a volar. Este instrumento es una adaptación creada en base a otros dos, el Cuestionario de Afrontamiento del Estrés de Sandín \& Chorot (2003) y el Inventario de Estrategias de Afrontamiento de Tobin, Holroyd, Reynolds \& Wigal (1989) en su adaptación al español por Cano, Rodríguez \& García (2007). Consta de 34 ítems, los cuales se responden en base a una escala Likert. La confiabilidad para esta muestra fue de .90 (Alfa).

- Escala de comportamientos aplacadores: Instrumento creado sobre la base de situaciones que comúnmente se viven dentro de un vuelo, que son realizados por el personal de la aerolínea, y que contribuyen a que éste sea menos estresante para los pasajeros. Consta de 10 ítems los cuales se responden de igual forma que los tres anteriormente detallados. Estas afirmaciones fueron sometidas a un procedimiento de validación de contenido y una aplicación piloto orientada a establecer su fiabilidad de base. La confiabilidad para esta muestra fue de .92 (alfa). 


\section{Resultados}

De un total de 287 personas, 238 manifestaron algún grado de miedo a volar en avión, lo que equivale al $82.9 \%$ de la muestra $(D T=3.7)$. El 17.1\% restante, que no presentó dicha característica, fue eliminado de los análisis posteriores.

Según la escala de miedo a volar, los participantes como grupo se ubicaron dentro del rango bajo con un promedio de 2.06, comparados con la media teórica de la escala (DT $\left.=1.01, M=3.0, t_{(237)}=-14.46, p=.001\right)$.
Al evaluar los aspectos hacia los cuales es probable presentar temor al volar, la afirmación "El avión vibra fuertemente por una turbulencia" se ubicó dentro del rango intermedio con un promedio de 3.21 siendo la que recibió mayor frecuencia de aceptación (47\%), seguida de "El avión acelera y despega" con un promedio de 2.58 (aceptación $=31.9 \%$ ). La situación a la que menos se le teme es "Oír hablar a otros sobre viajar en avión” ( $M=1.50$, aceptación $=4.8 \%)$, (Ver tabla 1$)$.

Tabla 1. Porcentajes de acuerdo en la escala Miedo a Volar.

\begin{tabular}{lccccc}
\hline \multicolumn{1}{c}{ Ítems } & $\begin{array}{c}\text { De } \\
\text { acuerdo }\end{array}$ & Neutro & $\begin{array}{c}\text { En } \\
\text { desacuerdo }\end{array}$ & Media & $\begin{array}{c}\text { Desviación } \\
\text { típica }\end{array}$ \\
\hline El avión vibra fuertemente por una turbulencia & 47.0 & 13.9 & 35.7 & 3.21 & 1.36 \\
El avión acelera y despega & 31.9 & 12.2 & 55.8 & 2.58 & 1.49 \\
El avión aumenta la altitud & 29.4 & 13.0 & 57.5 & 2.51 & 1.51 \\
El avión se mueve por las nubes y el viento & 26.9 & 13.9 & 59.2 & 2.47 & 1.45 \\
Oír la aceleración del motor & 25.2 & 14.3 & 60.5 & 2.38 & 1.41 \\
El avión empieza a descender & 24.3 & 16.0 & 59.7 & 2.37 & 1.39 \\
El avión toma la salida por la pista de despegue & 20.5 & 13.0 & 66.4 & 2.17 & 1.36 \\
El avión aterriza & 18.9 & 14.7 & 63.3 & 2.15 & 1.38 \\
Mirar por la ventana durante el vuelo & 18.5 & 15.1 & 66.4 & 2.08 & 1.38 \\
Esperar el momento de la salida & 18.5 & 10.9 & 70.6 & 2.00 & 1.35 \\
Estar sentado en el avión mientras todavía está & 17.2 & 12.6 & 70.1 & 2.03 & 1.33 \\
en tierra & & & & & \\
Entrar en el avión & 16.8 & 12.2 & 71.0 & 2.00 & 1.31 \\
Se cierran las puertas del avión & 16.0 & 13.0 & 71.0 & 1.97 & 1.31 \\
Decidir viajar en el avión & 13.5 & 8.0 & 78.6 & 1.74 & 1.28 \\
Planificar un viaje en avión & 12.6 & 7.1 & 80.3 & 1.73 & 1.21 \\
Ir hacia el aeropuerto & 12.2 & 13.4 & 74.3 & 1.83 & 1.28 \\
El avión pone el freno y reduce la velocidad & 11.8 & 13.9 & 74.3 & 1.87 & 1.26 \\
Llevar a otros al aeropuerto & 7.9 & 10.5 & 81.5 & 1.61 & 1.11 \\
Ver un avión en la televisión o en una película & 6.3 & 10.9 & 82.8 & 1.49 & 1.06 \\
Ver un avión en vuelo & 5.4 & 11.8 & 82.8 & 1.50 & 1.04 \\
Oír hablar a otros sobre viajar en avión & 4.6 & 1.8 & 83.6 & 1.50 & 1.00 \\
\hline
\end{tabular}

Según la escala de expectativas de peligro y ansiedad al volar, encontramos que la situación que genera mayor preocupación fue "Podría ponerme nervioso" $(M=2.71$; $D T=1.44)$, seguida de "El avión podría entrar en medio de una tormenta" $(M=2.59 ; D T=1.4)$. Para esta escala, la situación que genera menos ansiedad se refiere a que "El avión podría chocar con otro avión" $(M=1.70 ; D T=1.2)$ (Ver tabla 2). 
Tabla 2. Porcentajes de acuerdo afirmaciones Escala Expectativas de peligro y ansiedad al volar.

\begin{tabular}{|c|c|c|c|c|c|}
\hline Ítems & De acuerdo & Neutro & En desacuerdo & Media & $\begin{array}{l}\text { Desviación } \\
\text { típica }\end{array}$ \\
\hline Podría ponerse nervioso & 33.6 & 16.8 & 49.6 & 2.71 & 1.44 \\
\hline El avión podría entrar en medio de una tormenta & 29.5 & 19.4 & 51.1 & 2.59 & 1.34 \\
\hline Su corazón podría palpitar o latir muy rápido & 27.7 & 17.6 & 54.6 & 2.53 & 1.38 \\
\hline Usted podría sentir náuseas & 23.9 & 18.5 & 57.6 & 2.38 & 1.37 \\
\hline Su estómago podría retorcerse & 23.5 & 17.2 & 59.2 & 2.39 & 1.36 \\
\hline Usted podría sentirse mareado & 22.7 & 18.5 & 58.8 & 2.35 & 134 \\
\hline Uno de los motores podría incendiarse & 21.8 & 16.8 & 61.3 & 2.29 & 131 \\
\hline El avión podría caerse desde el cielo y estallar & 21.8 & 13.9 & 64.3 & 2.24 & 1.40 \\
\hline Podrían atascarse uno o más motores del avión & 20.6 & 14.3 & 65.1 & 2.23 & 1.31 \\
\hline $\begin{array}{l}\text { Usted podría ponerse a temblar o tener sensación de } \\
\text { inestabilidad }\end{array}$ & 19.3 & 13.0 & 67.6 & 2.14 & 1.32 \\
\hline $\begin{array}{l}\text { El tren de aterrizaje podría no funcionar en el mo- } \\
\text { mento del aterrizaje }\end{array}$ & 18.1 & 14.3 & 67.6 & 2.15 & 1.28 \\
\hline Usted podría sudar excesivamente & 16.0 & 17.6 & 66.4 & 2.09 & 1.28 \\
\hline $\begin{array}{l}\text { Podría experimentar sensaciones corporales poco } \\
\text { usuales }\end{array}$ & 16.0 & 17.6 & 66.4 & 2.08 & 1.29 \\
\hline Uno de los motores podría desprenderse & 15.5 & 11.8 & 72.7 & 1.96 & 1.26 \\
\hline El avión podría perder gasolina en medio del aire & 15.1 & 16.4 & 68.5 & 2.04 & 1.24 \\
\hline Podría tener dificultad para pensar con claridad & 15.1 & 16.4 & 68.5 & 2.07 & 1.26 \\
\hline Un ala podría caerse & 14.3 & 12.2 & 73.5 & 1.92 & 1.22 \\
\hline Podría perder el control de sus sentimientos & 13.1 & 12.2 & 74.7 & 1.86 & 1.22 \\
\hline El avión podría chocar con otro avión & 9.7 & 11.4 & 78.9 & 1.70 & 1.15 \\
\hline
\end{tabular}

$\mathrm{Al}$ evaluar las estrategias de afrontamiento que los participantes declaran utilizar, la que presenta mayor frecuencia es la estrategia de "Reevaluación Positiva" $(M=2.73 ; D T$ $=0.94)$, seguido de la "Evitación" $(M=2.8 ; D T=0.83)$. Luego se encuentra la estrategia de nombre "Focalizado en la solución de problemas" $(M=2.7 ; D T=1,01)$, seguido de "Religión" $(M=2.3 ; D T=0.8)$, "Autofocalización Negativa" $(M=2.4 ; D T=0.83)$ y "Apoyo Social" $(M=2.1$; $D T=0.83$ ). La que presentó un menor uso declarado fue la estrategia de "Expresión Emocional Abierta" $(M=1.7 ; D T$ $=0.7)$, (Ver tabla 3 ). 
Tabla 3: Porcentajes de acuerdo uso de Estrategias de Afrontamiento

\begin{tabular}{lccccc}
\hline \multicolumn{1}{c}{ Ítems } & De acuerdo & Neutro & En desacuerdo & Media & $\begin{array}{c}\text { Desviación } \\
\text { típica }\end{array}$ \\
\hline Reevaluación positiva & 40.4 & 10.9 & 48.7 & 2.73 & 0.94 \\
Evitación & 37.0 & 10.9 & 52.1 & 2.79 & 0.83 \\
Focalizado en la solución de proble- & 34.9 & 7.1 & 58.0 & 2.65 & 1.01 \\
mas & 25.3 & 7.1 & 67.6 & 2.28 & 1.13 \\
Religión & 14.7 & 14.3 & 71.0 & 2.36 & 0.79 \\
Autofocalización negativa & 12.6 & 9.2 & 78.2 & 2.09 & 0.83 \\
Búsqueda de apoyo social & 3.0 & 5.5 & 91.6 & 1.68 & 0.68 \\
Expresión emocional abierta & & &
\end{tabular}

Respecto a las situaciones que potencialmente podrían resultar aplacadoras del miedo a volar y que dependen de factores contextuales ajenos al individuo, los resultados indican que recibir información completa respecto a una situación particular, como el retraso de un vuelo o cambios en el clima, es la que más les facilita el enfrentamiento de la ansiedad por volar $(M=4.58$; DT $=0.8)$. En segundo lugar, encontramos "que en el caso de haber turbulencias, el capitán explique lo que está pasando y diga que no es peligroso" $(M=4.30 ; D T=0.8)$. La acción que es descrita como la menos útil es "que antes de subir al avión se dé información sobre el vuelo" $(M=3.7 ; D T=1.34)$, (Ver tabla 4).

Tabla 4: Porcentajes de acuerdo afirmaciones escala situaciones que ayudan.

\begin{tabular}{|c|c|c|c|c|c|}
\hline Ítems & Me ayuda & Indiferente & No me ayuda & Media & Desviación típica \\
\hline $\begin{array}{l}\text { Que en el caso de haber alguna situación } \\
\text { especial se dé información completa. }\end{array}$ & 89.9 & 6.7 & 3.4 & 4.58 & 0.77 \\
\hline $\begin{array}{l}\text { Que en el caso de haber turbulencias, el } \\
\text { capitán explique lo que está pasando y diga } \\
\text { que no es peligroso. }\end{array}$ & 83.7 & 10.5 & 5.9 & 4.30 & 0.92 \\
\hline $\begin{array}{l}\text { Que en el caso de haber turbulencias, el } \\
\text { personal de abordo se mantenga tranquilo. }\end{array}$ & 82.7 & 13.0 & 4.2 & 4.35 & 0.89 \\
\hline $\begin{array}{l}\text { Que el interior del avión se vea cuidado, sin } \\
\text { cosas rotas o sin funcionar. }\end{array}$ & 82.3 & 11.8 & 5.9 & 4.35 & 0.99 \\
\hline $\begin{array}{l}\text { Que el personal a bordo se muestre tranquilo } \\
\text { y alegre. }\end{array}$ & 77.7 & 18.1 & 4.2 & 4.29 & 0.96 \\
\hline $\begin{array}{l}\text { Que el capitán informe las condiciones } \\
\text { meteorológicas del vuelo. }\end{array}$ & 76.4 & 13.9 & 9.7 & 4.14 & 1.08 \\
\hline $\begin{array}{l}\text { Que el video de seguridad explique bien que } \\
\text { hacer en caso de peligro. }\end{array}$ & 73.9 & 16.4 & 9.7 & 4.12 & 1.09 \\
\hline $\begin{array}{l}\text { Que el folleto que el avión tiene explique } \\
\text { bien que hacer en caso de peligro. }\end{array}$ & 70.2 & 20.2 & 9.7 & 4.09 & 1.12 \\
\hline $\begin{array}{l}\text { Que el capitán se comunique con los } \\
\text { pasajeros durante el vuelo. }\end{array}$ & 66.8 & 22.3 & 10.9 & 3.90 & 1.15 \\
\hline $\begin{array}{l}\text { Que antes de subir al avión, den información } \\
\text { sobre el vuelo }\end{array}$ & 62.2 & 18.5 & 19.3 & 3.69 & 1.34 \\
\hline
\end{tabular}


Al analizar las técnicas de afrontamiento según género, se observan diferencias significativas únicamente para "Expresión Emocional" y para "Religión". En el caso de "Expresión Emocional", las mujeres $(M=1.76 ; D T=0.70)$ presentan un mayor promedio de adhesión a este tipo de estrategia que los hombres $(M=1.56 ; D T=0.64), t_{(236)}=2.29$, $p=.02$. En el caso de la estrategia "Religión" las mujeres $(M=2.45 ; D T=1.11)$ también presentan un promedio más elevado que los hombres $(M=2.00 ; D T=1.10), t_{(236)}=2.29, p$ $=.02$. Se observan diferencias marginalmente significativas en las estrategias de "Evitación" y "Revalidación Positiva", las mujeres $(M=2.86 ; D T=0.82)$ presentan un mayor uso de la evitación que los hombres $(M=2.67 ; D T=0.82)$, $t_{(236)}=1.77, p=.08$. Lo mismo ocurre con la revalidación positiva donde las mujeres $(M=2.82 ; D T=0.92)$ declaran utilizar más esta estrategia que los hombres $(M=2.60 ; D T$ $=0.97), t_{(236)}=1.75, p=.08$. No se observan diferencias no significativas ni para "Apoyo Social", ni "Solución de Problemas" ni "Autofocalización Negativa".

Al analizar la escala "Miedo a volar total" según género, se observan diferencias significativas en cuatro sub escalas. En todas ellas, las mujeres presentan promedios de temor mayores que los hombres. En primer lugar encontramos la situación "oír la aceleración del motor del avión", donde las mujeres $(M=2.52 ; D T=1.38)$ obtuvieron valores más elevados que los hombres $(M=2.15 ; D T=1.43), t_{(236)}=$ $-2.0, p=.04$. A continuación se encuentra "estar sentado en el avión mientras éste aún está en tierra", en donde las mujeres muestran puntajes de $(M=2.18 ; D T=1.35)$ y los hombres $(M=1.80 ; D T=1.29), t_{(236)}=-2.17, p=.03$, seguido de "esperar el momento de la salida" donde las mujeres $(M=$ 2.14; $D T=1.38)$ indican tener mayor temor que los hombres $(M=1.78 ; D T=1.29), t_{(236)}=-1.97, p=.05$. Finalmente, "se cierran las puertas del avión" provoca mayor miedo en las mujeres $(M=2.12 ; D T=1.32)$ que en los hombres $(M$ $=1.73 ; D T=1.27), t_{(236)}=-2.26, p=.02$. En resumen, las diferencias por género se manifiestan frente a un conjunto de situaciones, todas ellas asociadas a los momentos previos o iniciales del vuelo; en todas ellas, las mujeres manifiestan mayor temor que los hombres.

No se observaron diferencias significativas según género ni para la variable "Situaciones que ayudan" ni para "expectativas de peligro". Frente a la totalidad de las situaciones potencialmente aplacadoras del temor y a las expectativas de potenciales situaciones peligrosas, los participantes, tanto mujeres como hombres, opinaron de manera equivalente

Respecto a la variable edad, los análisis indican la existencia de correlaciones positivas entre dicha variable y las estrategias de afrontamiento:" Religión" $\left(r_{(\mathrm{n}=238)}=0.41\right.$, $p=.001)$, "Autofocalización Negativa" $\left(r_{(\mathrm{n}=238)}=0.27, p=\right.$ $.001)$, y "Reevaluación positiva" $\left(r_{(\mathrm{n}=238)} \stackrel{(\mathrm{n}=238}{=} 0.20, p=.01\right)$. Mientras mayor es la edad de la persona mayor es también el uso de dichas estrategias de afrontamiento y viceversa.

\section{Discusión}

El objetivo de este estudio fue profundizar en las características del miedo a volar, en particular, se midieron tanto las expectativa de peligro, las estrategias de afrontamiento más utilizadas y las situaciones que potencialmente facilitan la resolución de esta situación de estrés y que no dependen directamente del individuo.

Los resultados principales indican, en primer lugar, que más del $80 \%$ de los participantes manifiestan algún grado de miedo al momento de viajar en avión. Aunque los niveles generales son más bien bajos, y no corresponden a promedios atribuibles a la presencia de un miedo paralizante o fobia, sí es descrito como un fenómeno perturbador del bienestar. Este porcentaje es superior al reportado por la literatura y esta diferencia podría deberse a una serie de factores: En primer lugar, el aislamiento relativo de las personas que participaron en el estudio tiene como consecuencia que un porcentaje mayor al promedio nacional use este medio de transporte, sobre todo en situaciones de urgencia (cargadas de ansiedad) tales como problemas médicos, familiares, etc. En segundo lugar, la Patagonia austral es conocida por poseer un clima hostil que impone y determina la vida cotidiana de sus habitantes. En este sentido, algunos miedos atribuibles a la presencia potencial de turbulencia pueden relacionarse con los episodios cotidianos de vientos superiores a los $100 \mathrm{kms}$ por hora.

Las situaciones que más temor declaran despertar en las personas son las que se encuentran relacionadas con el movimiento del avión, como las turbulencias, el despegue, el aumento de altitud y el descenso. Esto probablemente se deba a que se trata de situaciones asociadas a la pérdida del control psicológico. Se trata de vivencias de cambio de estado que no dependen de la acción directa o indirecta de los individuos que la experimentan. Estos movimientos podrían representar simbólicamente un "viaje sin retorno" para las personas, donde cada proceso que se debe vivir en el avión altera su estabilidad emocional provocando ansiedad. Esto se puede relacionar con las expectativas de peligro que declararon las personas del estudio, donde se observa que sus miedos se enlazan a situaciones personales que potencialmente podrían salirse de control, es decir, tener miedo a ponerse nervioso, a que su corazón pueda acelerarse o a sentir náuseas. Estos resultados demuestran que las personas temen a una posible pérdida de control de lo que se está haciendo, en un escenario en donde se encuentran sometidos a las condiciones climáticas y de funcionamiento del avión. No obstante, lo anterior, los mecanismos de afrontamiento que las personas usan al viajar en avión se encuentran orientados a la "resolución de problemas" más que al "control de las emociones". Las más señaladas fueron "Reevaluación Positiva", seguido de "Evitación" y en tercer lugar "Focalizado en la solución de problemas", 
todas ellas clasificadas como estrategias de enfrentamiento y solución de los problemas. Una posible interpretación de estos datos es que las personas no poseen conciencia de que sus temores se encuentran vinculados al descontrol emocional, sin embargo, acuden a estrategias que van en una dirección inadecuada puesto que la situación que enfrentan no es posible de resolver por esta vía. Lo anterior podría conectarse con el uso habitual de tranquilizantes o similares para volar en avión. Si bien no contamos con resultados asociados a la frecuencia de uso de medicamentos de este tipo, resulta habitual, en la literatura de difusión, encontrar que este tipo de solución es elegida regularmente. Esta solución permitiría enfrentar las sensaciones físicas del miedo, anulándolas. En otros términos, las personas no parecen utilizar estrategias psicológicas de afrontamiento que sean de utilidad ya que no se encuentran focalizadas en el aspecto principal del temor a volar que parece encontrarse en el temor a perder el control emocional.

Desde una perspectiva de género las mujeres presentaron promedios de uso más elevados que los hombres en estrategias asociadas a estados emocionales internos, como "Expresión emocional" o "Religión". Estos datos apoyan estudios anteriores de Malcarne \& Fondacaro (1988) en donde manifestaban que las mujeres presentaban una tendencia a dar uso a EA que vinculaban sus emociones más que intentar solucionar problemas de manera directa o buscar distracciones. Siguiendo esta línea de pensamiento, las mujeres estarían utilizando estrategias más efectivas ya que como se menciona anteriormente, Magallanes es una zona en donde se debe poder convivir con un clima bastante hostil, especialmente referido a sus fuertes ráfagas de viento, y según esto sería más práctico intentar controlar las emociones que soslayar una realidad que se sabe es parte de la peculiaridad de esta región.

Con relación a los escenarios que son potencialmente aplacadores de la ansiedad, los participantes manifestaron que se sienten a gusto cuando el capitán o la tripulación dan una información completa frente a situaciones especiales, como por ejemplo frente a turbulencias o cambios de ruta. También señalan que les resulta útil ver al personal del avión en calma cuando se está en medio de turbulencias o cuando hay problemas con el clima. Como se expresa en otro apartado estas expectativas son más específicas y fáciles de delimitar (Tortella-Feliu \& Rubí, 2000), por lo cual las personas buscan de manera más veloz la forma de hacerles frente, que en este caso en particular tiene relación con el encontrar respuestas en profesionales que conocen más la situación que ellos mismos.

En resumen, la presente investigación buscó esclarecer las EA que las personas dicen utilizar frente al miedo a viajar en avión, sus temores, expectativas y situaciones que le permiten manejar mejor dicho temor. Los resulta- dos indican que los actuales programas disponibles para aprender a enfrentar el temor a volar se encuentran en la vía correcta ya que apuntan principalmente al control de las emociones. Si bien el estudio presenta limitaciones tanto por el tamaño de la muestra como por la ausencia de otras variables tanto demográficas como psicológicas que podrían enriquecer la comprensión del fenómeno, se trata de un estudio preliminar. Futuras investigaciones podrían realizarse en otros medios de trasporte similares en algunos aspectos (helicópteros, barcos, etc.), en las que también es posible observar temores y ansiedad del tipo estudiado. Aumentar el conocimiento de los procesos que acompañan el desarrollo y mantención de temores asociados a comportamientos cada vez más cotidianos, puede aportar tanto al mejoramiento de los programas de intervención existentes como a las políticas de las compañías aéreas.

\section{Referencias}

American Psychiatric Association (1995). Manual diagnóstico y estadístico de los trastornos mentales ( $4^{a}$ ed.). Barcelona: Masson. (Original de 1994.)

Bandura, A. \& Walters, R. (1974). Aprendizaje social y desarrollo de la personalidad. Madrid: Alianza Universidad.

Billings, A. G. \& Moos, R. H. (1981). The role of coping responses and social resources in attenuating the stress of Ufe events. Journal of Behavioral Medicine, 4(2), 139-57.

Bornas, X. \& Tortella-Feliu, M. (1995). Descripción y análisis psicométrico de un instrumento de autoinforme para la evaluación del miedo a volar. Psicología Conductual Revista Internacional de Psicología Clínica de la Salud, 3(1), 67-86.

Cano García, F.J., Rodríguez Franco, L. \& García Martínez, J. (2007). Adaptación española del Inventario de Estrategias de Afrontamiento. Actas Españolas de Psiquiatría, 35(1), 29-39.

Carretero, M. (1994). Constructivismo y Educación. Buenos Aires: Aique.

Cohen, S. \& Wills, T. A. (1985). Stress, Social Support, and the Buffering Hypothesis. Psychological Bulletin, 98(2), 310-357. http://dx.doi. org/10.1037/0033-2909.98.2.310

Cohen, S., Sherrod, D. \& Clark, M. (1986). The role of personality in the relationship between social support and well-being. Journal of Personality and Social Psychology, 50(5), 963-973.

Compas, B. E., Malcarne, V. \& Fondacaro, K. (1988). Coping with stressful events in older children and young adolescents. Journal of Consulting and Clinical Psychology, 56, 405-411.

Contini, N., Coronel, P., Levin, M. \& Estevez, A. (2003). Estrategias de afrontamiento y bienestar psicológico en adolescentes escolarizados de Tucumán. Revista de Psicología, 21(1), 179-200. Recuperado de http://revistas.pucp.edu.pe/index.php/psicologia/article/view/3723.

Cummings, T.N. (1989). Flying phobia. En C. Lindemann (dir.), Handbook of phobia therapy: rapid syntom relieve in anxiety disorders. Northvale, NY: Aronson. 
Curtis, G. C., Magee, W. J., Eaton, W.W., Wittchen, H.U. \& Kessler, R.C., (1998). Specific fears and phobias: Epidemiology and classification. British Journal of Psychiatry, 173, 212-217.

Darwin, C. (1872). The expression of emotion in men and animals. Londres: Murray.

Endler, N. S. \& Parker, J. (1990). Multidimensional assessment of coping: A critical evaluation. Journal of Personality and Social Psychology, 58(5), 844-854. DOI: 10.1037//0022-3514.58.5.844.

Escamilla, M., Rodríguez, I. \& González, G. (2009). El estrés como amenaza y como reto: un análisis de su relación. Ciencia y Trabajo, 32, 96-101.

Gonzalez-Celis, A. \& Padilla, A. (2006). Calidad de vida y estrategias de afrontamiento ante problemas y enfermedades en acianos de cuidad de México. México: Universidad Autónoma de México.

Gray, J. A. (1971). La psicología del miedo. Guadarrama: Madrid.

Greco, T. S. (1989). A cognitive behavioral approach to fear of flying: a practitioner's guide. Phobia Practice and Research Journal, 2(1), 3-15.

Gursky, D. M. \& Reiss, S. (1987). Identifying danger and anxiety expectancies as components of common fears. Journal of Behavior Therapy and Experimental Psychiatry, 18(4), 317-324. doi:10.1016/00057916(87)90045-0

Howard, W.A., Murphy, S.M. \& Clarke, J.C., (1983). The nature and treatment of fear of flying: a controlled investigation. Behavior Therapy, 14(4), 557-567.

Krzemien, D., Urquijo, S. \& Monchietti, A. (2004). Psicothema: Aprendizaje social y estrategias de afrontamiento a los sucesos críticos del envejecimiento femenino. Buenos Aires: Universidad Nacional de Mar del Plata.

Lazarus, R.S. \& Folkman S. (1986). Estrés y procesos cognitivos. Barcelona: Martínez Roca.

Lazarus, R.S. (1994). Passion and reason: Making sense of our emotions. New York: Oxford University Press.

Malcarne, V. \& Fondacaro, K. (1988). Coping with stressful events in older children and young adolescents. Journal of Consulting and Clinical Psychology, 55, 534-541.

McCrae, R. \& Costa, P.T. (1986). Personality, coping and coping effectiveness in adult sample. Journal of Personality, 54, 385-405. doi:10.1111/j.1467-6494.1986.tb00401.x

Reiss, S. (1980). Pavlovian conditioning and human fear: An expectancy model. Behavior Therapy. 11(3), 380-396.

Roger, D., Jarvis, G. \& Najarían, B. (1993). Detachment and coping: The construction and validation of a new scale for measuring Coping strategies. Personality and Individual differences 15, 619-626.

Roussi, P. \& Vassilaki, E. (2001). The applicability of the multiaxial model of coping to a greek population. Anxiety, Stress and Coping, 14(2), 125-147.

Sandín, B. \& Chorot, P. (2003). Screening del cáncer de mama. Afectación psicológica. Madrid: Colección Ciencias de la Salud, UNED.

San Martín, C. (2012). Las representaciones sociales de la seguridad ciudadana en los vecinos de la comuna de Melipilla, Chile. Psicoperspectivas, 12(1), 71-94.

Sosa, C. D., Capafóns, J. I. \& Viña, C., (1993). Estudio descriptivo de la fobia a volar a través de la IDG-FV. Comunicación presentada al XXIV Congreso iberoamericano de psicología, Santiago de Chile.

Sosa, C.D., Capafóns, J.I., Viña, C.M. \& Herrero, M. (1995). La evaluación del miedo a viajar en avión: estudio psicométrico de dos instrumentos de tipo autoinforme. Psicología Conductual, 3, 133-158.

Tobin, D.L., Holroyd, K.A., Reynolds, R.V. \& Wigal, J.K. (1989). The hierarchical factor structure of Coping Strategies Inventory. Cognitive Therapy and Research, 13(4), 343-361.

Tortella-Feliu, M. \& Rubí, A. (2000). Las expectativas de peligro como componente del miedo a volar en avión. España: Universidad de Madrid.

Van Gerwen, L. J., Spinhoven, R., Diekstra, R. F. \& Van Dyck, R. (1997). People who seek help for fear of flying: typology of flying phobics. Behavior Therapy, 28, 237-251.

Vázquez, C., Crespo, M. \& Ring, J. (2003). Clínica en Psiquiatría y Psicología, capítulo 31: Estrategias de afrontamiento. Barcelona: Elsevier-Masson. 